\title{
BIOMECHANICAL AND FRACTAL ANALYSIS OF ULNA BONE
}

\section{FRANCISCO CASANOVA-DEL-ANGEL}

Polytechnic Institute National. Mexico

Juan de Dios Bátiz S/N

Unit. Adolfo López Mateos

07738 Ciudad de Mexico

Mexico

e-mail: fcasanova@ipn.mx

\begin{abstract}
This article provides a geometrical study of fractal direction in bones, particularly on a cubitus, based on biomechanical concepts of fractal analysis. First, generating fractal curve is defined and, based on that, elliptical fractal curve. Then propagation direction of the latter is built. Application is then carried out based on the study of a real fracture of ulna bone, under the analysis of mechanical parameters of the bone, in order to obtain the fractal dimension of every stroke and status of the fracture. Methodology developed shows the behaviour of cinematic and mechanical phenomena in living beings considered as complex systems made by tissues, solids, and mechanical bodies.
\end{abstract}

\section{Introduction}

To begin with, it must be taken into account that biological structures are being used, which may be geometrically built with simple shapes (straight lines, circles, spheres, ellipses, and polygons), and that a high number of their structural properties (length, area, volume, hardness,

Keywords and phrases: elliptic fractal fracture, crack propagation, bones, biomechanics, fractal dimension.

Received March 3, 2016

(C) 2016 Scientific Advances Publishers 
compressive strength, stress, and moment of inertia of transversal section of the bone, among many others) may be estimated. It must also be remembered that bones are classified in accordance with their shape: long, short, flat or irregular. Every bone in the skeleton has a dense external layer that seems to be soft and solid at first sight. The external layer is called compact bone and surrounds cancellous bone, a sort of honeycomb of small pieces that resemble needles or beams called trabecula's. Inside this shell is the medulla.

Regarding the bone under study, it must be considered that the ulna or cubitus is a long bone, parallel to radius, between the humeral trochlea and the carpus. It has a body and two extremities. It is located in the inner part of the arm; it articulates with humerus and radius on the top and with the radius and carpus bones on the bottom; it is not straight, it shows a slight anterior concave curvature; it is not circular, since it is in fact ellipsoid; it has an italic $S$ shape in a vertical transversal plane, concave inwards on the top and outwards on the bottom; it has more volume upwards than downwards; it is prismatic triangular in its three upper fourths and is regularly ellipsoidal in its lower fourth; it has three faces (anterior, posterior, and medial) and three margins (anterior, posterior, and lateral).

For a long time, medical-biological research has been needed to build mathematical models of biological and bone structures in their area of study. These are not compatible with conventional analyses in their field. It has also been realized that, if the biological system is capable of living with new final values, it means that it has adapted itself to new circumstances and it will evolve satisfactorily.

Among the reviewed documents on the topic, biomechanics and bones (1): basic concepts and classical mechanic essays [1] may be mentioned, when the principals of classical mechanics are applied to the study of the resistance of materials to fracture when subject to a certain load. The objective of this work is to describe the main concepts and fundamental 
principles used in biomechanics, focused on their application to bone tissue. In study called Relationship between morphostructural variables of fractal dimension, lacunarity, and entropy in trabecular bone of proximal portion of femur [2], fractal dimension (box-counting method), lacunatity (sliding box-method) and entropy properties (Grey level cooccurrence matrix) were calculated in three regions of the proximal femur in a sample of $20 \mathrm{X}$-ray images in anteroposterior projections. Significant strong inverse correlations were found between the fractal dimension and the lacunarity in all regions and between the entropy and the fractal dimension and lacunarity in proximal shape region. In The fractal dimension correlated to the bone mineral density [3]. After an introduction to the theory and fractal dimension, the box counting method was used for the segmentation of radiographic images, the study of the influence of range size boxes on the fractal dimension will be investigated, and the correlation between a reference dimension and bone mineral density. Other imaging techniques will be given in order to see the results of the application of the method on these types of images.

The focus in Fractal analysis of bone cell syncytium in normal diseased bone was to quantify the changes in interconnectedness and geometrical complexity in micrographs of bone from patients with bone diseased. The fractal dimension of the lacuna canalicular network were calculated and the applicability of fractal dimension as a diagnostic tool in orthopaedics was investigated [4]. The observation of trabecular changes of the mandible after orthognathic surgery using fractal analysis sought to evaluate trabecular changes in the mandible using fractal analysis and to explain the transient osteopenia related to rapid orthodontic tooth movement after orthognathic surgery, and found that bone density decreases after orthognathic surgery due to transient osteopenia related to the regional acceleratory phenomenon. This result can provide a guide to evaluating orthodontic tooth movement after orthognathic surgery [5]. 


\section{Definition of Generating Fractal Curve}

Definition of generator. Let $I_{0}$ be a single curved line segment, contained in a closed interval, that is, $I_{0} \subset[a, c]$. Let $I_{1}$ be a set with sectioned behaviour, consisting in three segments of a curved line which create, based on starting point $a$ of $I_{1}$, two triangles reflected regarding the point $b$ of $I_{1}$ such that $b \in[a, c]$ obtained as follows: the two first thirds of $I_{1}$ are substituted or removed by the sides of triangle which create an angle with $I_{0}$. This process is repeated for the third, but with the sides reflected from point $b$. Particularity of these triangles is that such are scalene or obtuse angle (without equal sides), where the joining point of triangles $b$ is not medium point of $I_{0}$, Figure 1(a).

This process is known as fractal curve generating which is called state 1 . Construction of set $I_{2}$ is made applying the generator to every segment of $I_{1}$, which is called state 2 . Thus, set $I_{k}$ is created applying generator $I_{1}$ on every segment of $I_{k-1}$, which is called state $k$, Figure $1(b)$.

Some particular aspects presented in the construction of this type of fractal curve should be noticed when generator is created by combination or triangular layouts (in size and form):

- States $I_{k-1}$ and $I_{k}$ are different from each other in the sequence shown by polygonal curves $\forall k \rightarrow \infty$.

- Set $I$ has a fine structure, that is, it contains every detail in every arbitrary small scale.

- Although the generator is composed by two triangular figures corresponding to Euclidean logic, the geometry of $I_{k} \forall k \rightarrow \infty$ is too irregular to be described in classical geometric terms.

Figure 1(b) shows the curve built on the plane through reflection of $I_{k}$ on every side. Figure 1(c) shows behaviour of status $I_{4}$ in an ellipse. 


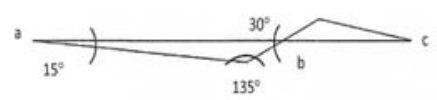

a)
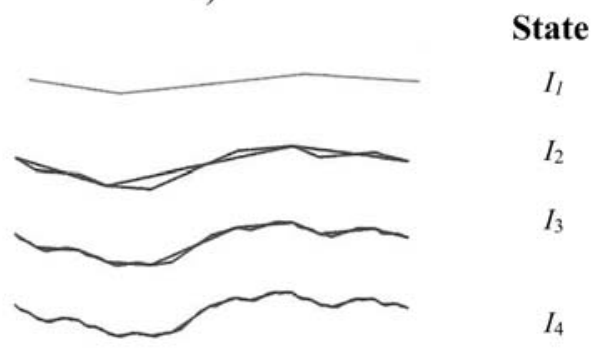

b)

c)

Figure 1. (a) Construction of the fractal curve $I$, (b) in every state $I_{k}$ generator $I_{1}$ is applied on every segment of the curve, and (c) ellipsoidal fractal curve.

The above was for the definition of a fractal generator on the plane. Let us now see what happens when the path of the fractal is curve. That is, let us consider a defined fractal function on points of a curve in space.

Definition of fractal curve. A set $I_{k} \subset \mathfrak{R}^{n}, \forall n=2,3$ and $k=0$, $1, \ldots$ is a continuous and simple section fractal curve (class $C^{1}$ in every section), if there is an injective function $f:[a, b] \rightarrow \mathfrak{R}^{n}$ (curve does not stop itself), regular such that $I_{k}=f([a, b])$.

As it is well known, a function of this type of curves is called parametrization of $I$, and points $f(a)$ and $f(b)$ are known as ends. In addition, the use of parametrizations to represent curves allows the use of calculation techniques with which properties of curves from functions defined along any curves are deduced. Introducing here the above definition of fractal curve, we may see that infinite and different parametrizations may be also admitted here. Some of these parametrizations are equivalence, since $f:[a, b] \rightarrow \mathfrak{R}^{n}$ and $g:[c, d] \rightarrow \mathfrak{R}^{n}$ two parametrizations of the same fractal curve, $\exists$ a bijective function 
$\vartheta:[a, b] \rightarrow[c, d]$ class $C^{1}$ such that $\vartheta^{\prime}(t) \neq 0, \forall t \in[a, b]$, such that $f=g$ or $\vartheta . \vartheta$ is called change of parameter and $f$ and $g$ are implied as equivalent. When parametrization of a fractal curve is chosen, the path direction is automatically defined for $I$, starting in $f(a)$ and ending in $f(b)$. Let us now see the definition of path direction for a fractal curve.

Definition of path direction. Let $f$ and $g$ be two parametrizations, the path direction of them is defined for $I$ if and only if the function for change of parameter is strictly increasing, that is, $\vartheta^{\prime}(t)>0, \forall t \in[a, b]$.

From the last definition, we may see that two more definitions may be included: reversed or opposite direction $\left(\vartheta^{\prime}(t)<0, \forall t \in[a, b]\right)$, and parametrizations crossed direction $\left(\vartheta^{\prime}(t)=0, \forall t \in[a, b]\right)$, parametrizations with a point in common. It is also considered that a fractal curve is oriented if a path direction is chosen for $I$.

Definition one of fractal curve orientation. With a given path direction of a fractal curve, showing that direction as $I^{+}, f$ is a parametrization of $I^{+}$if it is a parametrization of $I$ along such on the path direction defined for $I^{+}$.

Definition two of fractal curve orientation. With given end points $f(a)$ and $f(b)$ of parametrization $f$, orientation of such is given when the starting end point and the final end point are defined.

From here, if $g$ is another parametrization of $I$, it is said that it describes a fractal curve with opposite orientation, shown as $I^{-}$, if it is a parametrization of $I$ along such on opposite direction to defined orientation for $I^{+}$. In addition, if it is true that $g(t)=f(a+b-t)$, then we have an opposite parametrization. However, the case we are developing includes fractal curves ending or concurring on the same point or end point (or may start on the same point). Therefore, let two open fractal curves $I_{1}$ and $I_{2}$ such that $f:[a, b] \rightarrow \mathfrak{R}^{n}$ and $g:[c, b] \rightarrow \mathfrak{R}^{n}$, which are continuous section injective parametrizations, of $I_{1}$ and $I_{2}$, respectively, Figures 2(a) and 2(b). 


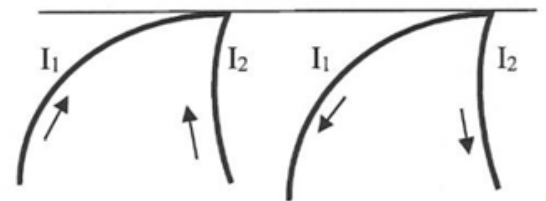

a)

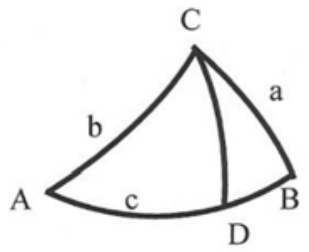

c)

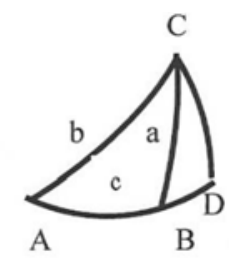

d)

Figure 2. Fractal curves: (a) positive orientation, (b) negative orientation, (c) spherical triangle where arch $C D$ falls over $\operatorname{arch} A B$, and (d) spherical triangle where arch $C D$ falls on extension of $\operatorname{arch} A B$.

Fractal curves of this type have the following parametrizations: $f:[a, b] \rightarrow \mathfrak{R}^{n}$ and $g:[c, b] \rightarrow \mathfrak{R}^{n}$ injective and continuous section, such that $f(a) \neq g(c)$ and $f(b)=g(b)$. Since, in practical terms, it is not easy to find these type of fractal curves, let us see the following theoretical approach. Let $U I_{k}^{i}$ be a finite joint of concurring fractal curves such that

$$
\cup I_{k}^{i}=I_{k}^{1}, \ldots, I_{k}^{i}, \quad \forall i=1, \ldots, n \& k=1,2,3 .
$$

Complying with the fact that their end point is the same, without any other point in common. Fractal curves defined by Equation (1) have significant properties such as: in every arch $I_{k}$ all the parametrizations are equivalent; when a section of fractal curve is oriented, the remaining ones are automatically oriented in a consecutive manner and, of course, care must be taken in order for the orientation of the set to be correct. It may be seen that closed fractal curves may be easily obtained from here.

\subsection{Elliptical curves}

Below, we shall work with elliptic curves, with the feature that one of them will be contained in the other one, Figure 3. Since inertia of a rotating object is determined by its moment of inertia, this being the resistance a rotating object opposes to change of its spinning speed, and 
considering bone as a hollow and semi-flattened cylinder, the moment of inertia of the elliptic transversal section is calculated through Equation (2):

$$
I_{y_{1}}=\frac{\pi}{64}\left[\left(x_{1} y_{1}^{3}\right)-\left(x_{2} y_{2}^{3}\right)\right] .
$$

With $x_{1}$ as the highest external diameter of transversal section in the point of application of force, $y_{1}$ the highest external diameter, as well as $x_{2}$ and $y_{2}$ being the internal diameters of transversal section.

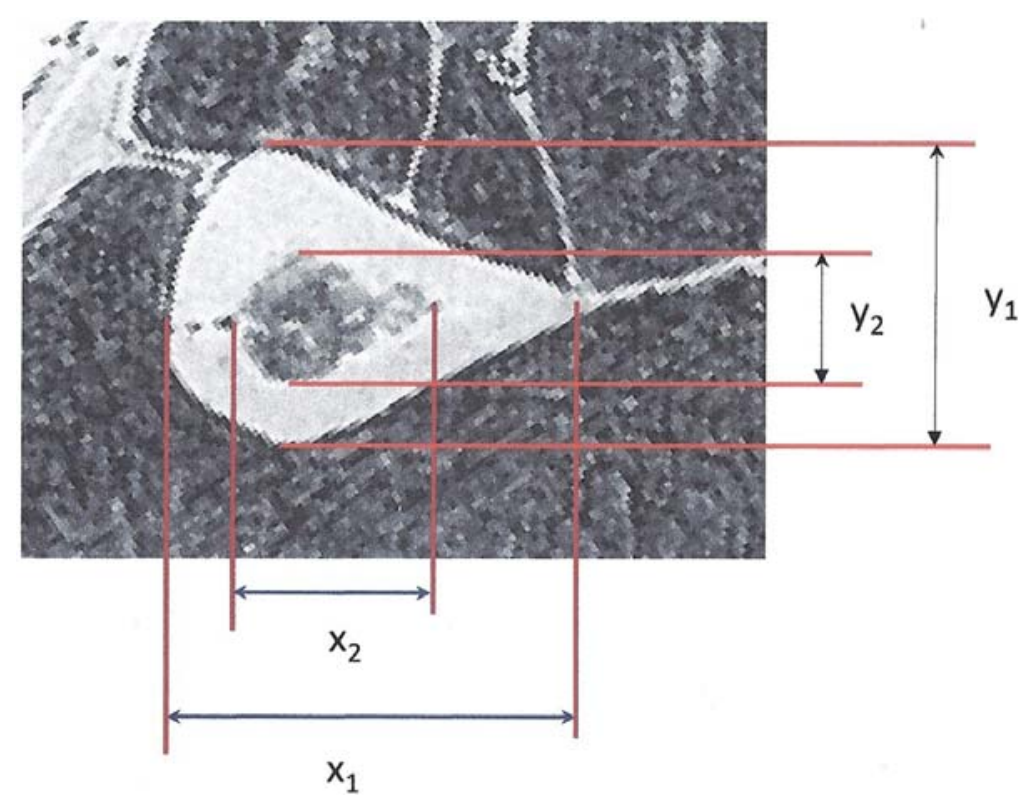

Figure 3. Transversal section of a transversal of an ulna bone, where $\left(x_{1}, y_{1}\right)$ and $\left(x_{2}, y_{2}\right)$ are diameters of the two ellipses.

\subsection{Propagation direction of a fractal curve and its characterization}

Now, in order to calculate the propagation direction of a curve fracture, we must take into account that when a trihedron angle has its vertex in the center of a sphere, its intersection with curved surface is a spherical angle. For specific reasons, we will only talk about oblique- 
angles, where the fact that a spherical triangle is the spherical surface obtained through intersection of the sphere and a trihedron which vertex is its center is complied with. Let $A B C$ be a spherical triangle and $C D$ an arch perpendicular to $A B$. Based on the above, we have two cases: (i) that $C D$ falls on $A B$, Figure 2(c), and (ii) that $C D$ falls on its extension, Figure $2(d),[7,8]$.

Proposition 1. In terms of sides $a, b$, and $c$, with $s$ being half of the addition of the arches or sides of the spherical triangle, that is, $s=\frac{a+b+c}{2}$, tangents of its semi-angles are given by Equations (3.1), (3.2), and (3.3):

$$
\begin{aligned}
& \operatorname{tg} \frac{A}{2}=\sqrt{\frac{\operatorname{sen}(s-b) \operatorname{sen}(s-c)}{\operatorname{sen} s \operatorname{sen}(s-a)},} \\
& \operatorname{tg} \frac{B}{2}=\sqrt{\frac{\operatorname{sen}(s-c) \operatorname{sen}(s-a)}{\operatorname{sen} s \operatorname{sen}(s-b)},} \\
& \operatorname{tg} \frac{c}{2}=\sqrt{\frac{\operatorname{sen}(s-a) \operatorname{sen}(s-b)}{\operatorname{sen} s \operatorname{sen}(s-c)} .}
\end{aligned}
$$

Demonstration. Based on trigonometry, it is known that: $\sin b$ $\sin c \cos A=\cos a-\cos b \cos c, \quad$ therefore: $\quad \cos A=\frac{\cos a-\cos b \cos c}{\operatorname{sen} b \operatorname{sen} c}$. Subtracting 1 to both members of the above equation, we have: $1-\cos A$ $=1-\frac{\cos a-\cos b \cos c}{\operatorname{sen} b \operatorname{sen} c}=\frac{\cos b \cos c+\operatorname{sen} b \operatorname{sen} c-\cos a}{\operatorname{sen} b \operatorname{sen} c}$. So: $2 \operatorname{sen}^{2} \frac{A}{2}=$ $\frac{\cos (b-c)-\cos a}{\operatorname{sen} b \operatorname{sen} c}$, but: $\cos y-\cos x=2 \operatorname{sen} \frac{x+y}{2} \operatorname{sen} \frac{x-y}{2}$, then: $\operatorname{sen}^{2}$ $\frac{A}{2}=\frac{\operatorname{sen} \frac{a+(b-c)}{2} \operatorname{sen} \frac{a-(b-c)}{2}}{\operatorname{sen} b \operatorname{sen} c}$.

For practical and presentation reasons, side relationships may be expressed as follows:

$$
\begin{aligned}
& a+b-c=(a+b+c)-2 c=2 s-2 c=2(s-c), \\
& a-b+c=(a+b+c)-2 b=2 s-2 b=2(s-b) .
\end{aligned}
$$


Which implies that sinuses of the semi-angle for every vortex may be expressed in accordance with (3.4):

$$
\begin{gathered}
\operatorname{sen} \frac{A}{2}=\sqrt{\frac{\operatorname{sen}(s-b) \operatorname{sen}(s-c)}{\operatorname{sen} b \operatorname{sen} c}}, \quad \operatorname{sen} \frac{B}{2}=\sqrt{\frac{\operatorname{sen}(s-c) \operatorname{sen}(s-a)}{\operatorname{sen} c \operatorname{sen} a}}, \\
\operatorname{sen} \frac{C}{2}=\sqrt{\frac{\operatorname{sen}(s-a) \operatorname{sen}(s-b)}{\operatorname{sen} a \operatorname{sen} b}}
\end{gathered}
$$

Developing the same procedure, but now for cosinus of the angle, we obtain (3.5):

$$
\begin{gathered}
\cos \frac{A}{2}=\sqrt{\frac{\operatorname{sen} s \operatorname{sen}(s-a)}{\operatorname{sen} b \operatorname{sen} c}}, \cos \frac{B}{2}=\sqrt{\frac{\operatorname{sen} s \operatorname{sen}(s-b)}{\operatorname{sen} c \operatorname{sen} a}}, \\
\cos \frac{C}{2}=\sqrt{\frac{\operatorname{sen} \operatorname{sen}(s-c)}{\operatorname{sen} a \operatorname{sen} b}}
\end{gathered}
$$

At last, dividing every equation of (3.5) by (3.4), we obtain (3.1), (3.2), and (3.3).

\section{QED}

Fractal curve $I$ is characterized by being of a similar scale, based on a transformation $F: \mathfrak{R}^{n} \rightarrow \mathfrak{R}^{n}$ with $\lambda_{i}>0 \forall i \exists_{n} a, b \in \mathfrak{R}^{n}$ such that $\left|F_{i}(a)-F_{i}(b)\right|=\lambda_{i}|a-b|$. Similarity of scale is present for triangles created with $I_{0}$, by generator $I_{1}$. In a like manner, it also has the property to be affine, since it is, based on transformation $F$ already defined, $F(a)=T(a)+\alpha$ with $T$ a non-singular linear transformation and $\alpha \in \mathfrak{R}^{n}$. It must be remembered that affinity is conceived of as a shear transformation or as resistant to cutting, and is a contracting or expanding effect, not necessarily in the same direction.

A curve type $l_{k}$ satisfies the scale principle if all its relative figures are linked to each other by a scale law, and the property of inverse scale is complied with. 


\subsection{Meshing and definition of fractal outline}

Let $N\left(t^{*}, \Delta t^{*}\right)$ be the number of squares contained by reticulate, and $N(t, \Delta t)$ the number of squares intersected by the fractal curve. $D_{\theta^{\circ}}(I)$ shall be the fractal dimension, $\mathcal{L}$ the total length of the object, and $\mathcal{C}$ the length of every segment. Therefore, $\mathcal{L} / \mathcal{l}$ quotient defines the number of subdivisions contained by every side of the intersected reticulate. These scale properties correspond to a fragmented fractal [8, 9], and the multifractal [10], pp. 45 concept is applied. Based on the above

$$
N\left(t^{*}, \Delta t^{*}\right) \cong \Delta t^{*} \rho\left(t^{*}\right) t^{f\left(t^{*}\right)},
$$

where $t \in\left[t^{*}, t^{*}+\Delta t^{*}\right] . P\left(t^{*}\right)$ is the probability of distribution of intersection points $t \in\left[t^{*}, t^{*}+\Delta t^{*}\right]$ and $f\left(t^{*}\right)$ the fractal dimension of such points.

Considering a random generation of $f(t)$, the original curve is rotated to different angles, preferably constant, in order to calculate $D_{\theta^{\circ}}$ for every case

$$
D_{\theta^{\circ}}=\frac{\sum_{i}\left\{D_{\theta^{\circ} i} \forall i=1, \ldots, n\right\}}{n} .
$$

In order to rotate the original curve a certain number of times, let us consider mapping $M_{n}: \chi \rightarrow \mathfrak{R}$, where $M_{n}(t)=-\log \mu\left[B_{\delta n}(t)\right], \quad$ if $\mu\left[B_{\delta n}(t)\right]>0$, then $C_{n}(t)$ is a re-escalated version of $M_{n}(t)$, that is,

$$
C_{n}(t)=\frac{M_{n}(t)}{-\log \left(\delta_{n}\right)}
$$

where $C_{n}$ describes the local behaviour of $\mu$ measurement.

\subsection{Behaviour pattern}

The fractal behaviour of a geometrical discontinuity takes us to the concept of diagonal self-affinity. In order to define the pattern of the fractal generator, let us begin drawing straight lines from its base to the points where such curves go all along its length, thus obtaining a 
fractioned curve. It is useful to draw horizontal lines in case there is a change in its path behaviour, in order to identify the affinity along such a path.

Horizontal lines identifying the beginning of the generator must show the feature of proportionality $d$ such that: $d=\mathcal{L} \sin \beta$, where $\sin \beta=\frac{d}{\mathcal{L}}$ and $\cos \beta=\frac{k}{\mathcal{L}} \cdot \mathcal{L}$ is the length of the generator, $\mathcal{l}$ the length of every segment, and $\mathcal{L} / \mathcal{L}$ is the number of subdivisions contained by the generator. In order to observe the ideal behaviour of the path, the scale relationship is defined as the average of the lengths of adjoining generators, that is, $\left(\mathcal{L}_{1}+\mathcal{L}_{2}\right) / 2$; Figure $4(\mathrm{a})$. The scale factor of every rotation undergone by the generator or fractal curve, $s$, is defined as: $s=\log _{10}(\mathcal{N}) \quad \forall \mathcal{N} \rightarrow \infty$, where the value of $\mathcal{N}$ is the average of the highest number of every rotation, that is: $\mathcal{N}=\sum_{i}\left\{R_{i} \quad \forall b\right.$ and $\left.i=1, \ldots, n\right\}$. Based on the above, we can get the scale factor of the generator, defined as the inversion of $s$, which applied, generates the geometrical structure.

\subsection{Parameters of fractal geometry}

Given the fact that determining the real length of a fractal line depends on accurateness of measurement and is an important issue, the real total length of the line increases in accordance with equation number (6):

$$
\mathcal{L}_{j+1}=\mathcal{L}_{j} * \mathcal{N} * n
$$

With $j$ as number of iterations, $\mathcal{N}$ the number of elements in the base generator $(j=1)$ and $n$ the factor of reduction applied. Therefore, the real length of a fractal line $\left(\mathcal{L}_{j}\right)$ after $j$ iterations is given by equation number (7):

$$
\mathcal{L}_{j}=\mathcal{L}_{p} * \varepsilon_{j}^{\left(d-D^{*}\right)}
$$

where $\varepsilon_{j}=n^{j}$ represents the lowest measurement unit in order to measure the relative length of a segment of the fractal line. Parameter $d$ 
represents the Euclidian dimension of the object, $D^{*}$ is the fraction of the fractal dimension $D_{\theta^{\circ}}$ and $\mathcal{L}_{p}$ is the length of the whole fractal, from one end to the other, without taking into consideration its roughness. In order to apply the above to fractals generated from a natural process, $\varepsilon_{j}$ is cleared up from equation number (7), thus obtaining (8):

$$
\varepsilon_{j}=\left(d-D^{*}\right) \sqrt{\frac{\mathcal{L}_{j}}{\mathcal{L}_{p}}} .
$$

Property of axial symmetry of generator. Triangles creating segments of $I_{1}$, based on $I_{0}$ may vary regarding size and shape.

Generator $I_{1}$ may be considered as the union of two scalene triangles $I I_{1}$ (triangle lower than $I_{0}$ ) and $I I_{2}$ (triangle higher than $I_{0}$ ) created based on $I_{0}$, and reflected on $1 / 2 I_{0}$, that is,

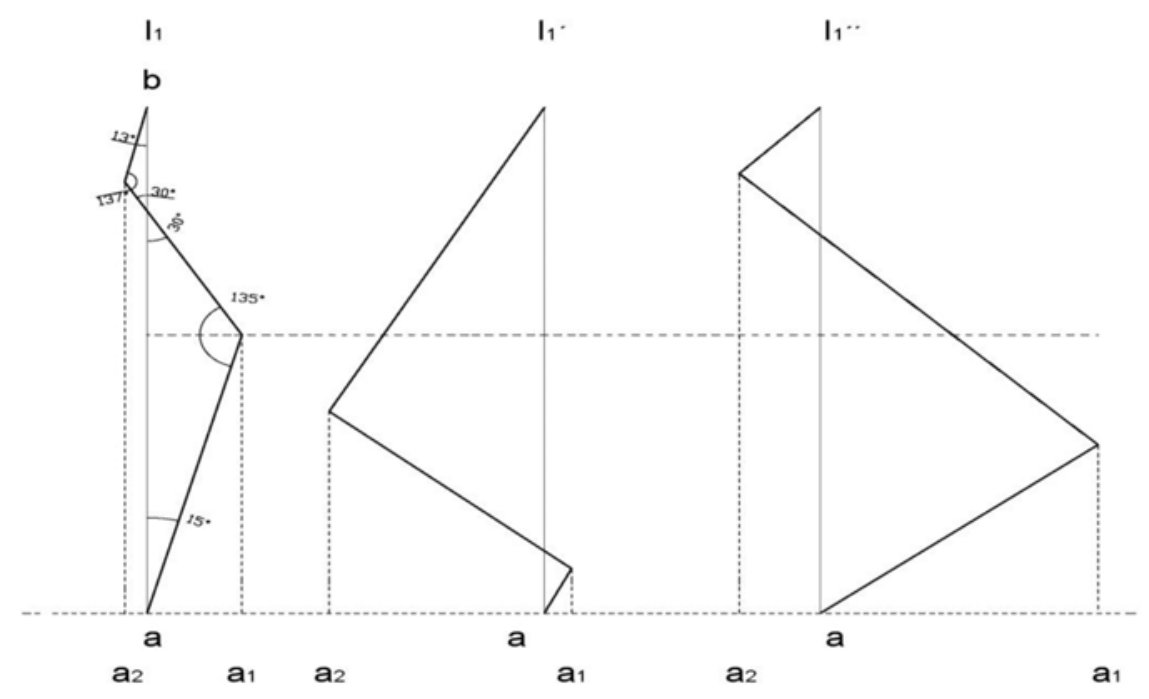

(a)

(b)

Figure 4. (a) Ideal generator, (b) affinity of triangles in the generator. 
When $\mathrm{z}_{1}=1 / 3 I_{0} ; \mathrm{z}_{2}=1 / 2 I_{0} \quad$ and $\quad \mathrm{z}_{3}=2 / 3 I_{0} \quad$ we talk about generator $I_{1}$, Figure 4(b) left. When $\mathrm{z}_{1}<1 / 3 I_{0} ; \mathrm{z}_{2}<1 / 2 I_{0}$ and $\mathrm{z}_{3}<2 / 3 I_{0}$, we talk about $I_{1}^{\prime}$, Figure $4(\mathrm{~b})$ right. When $\mathrm{z}_{1}>1 / 3 I_{0} ; \mathrm{z}_{2}>$ $1 / 2 I_{0}$ and $\mathrm{z}_{3}>2 / 3 I_{0}$, we talk about $I_{1}^{\prime \prime}$. In the two latter cases, the homologous angles creating the segments of the generator are congruent and their homologous sides are proportional, thus complying with the property of similarity of triangles, that is, $I_{1}^{\prime}$ and $I_{1}^{\prime \prime}$ are generators similar to $I_{0}$.

In our case, the Theorem of transformation of linear affinity, is valid, which considers that if $G$ is a geometrical transformation of scale factors $r, s \in \mathfrak{R}$, such that $G: \mathfrak{R}^{n} \rightarrow \mathfrak{R}^{n}$, there are translation factors $h, k \in \mathfrak{R}$, which go back to $I_{m+1}$ an affine generator of $I_{m}$, see [11].

\section{Fractal in Bones}

Let us now see an application of the theory developed on curve fractals generated in an ulna fracture. Since we do not have the original bone (since it is still inside its owner), let us start with the flat view of the radiographic image in Figure 5(a); not models of bones carried out model breaking strength as a function of mineral density of bone. Density of bone is related to breaking strength and there are biological variations in breaking strength in patients with the same bone density. 


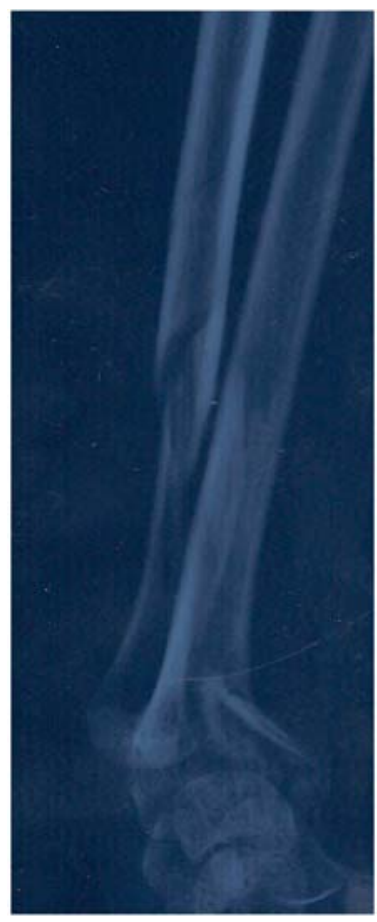

(a)

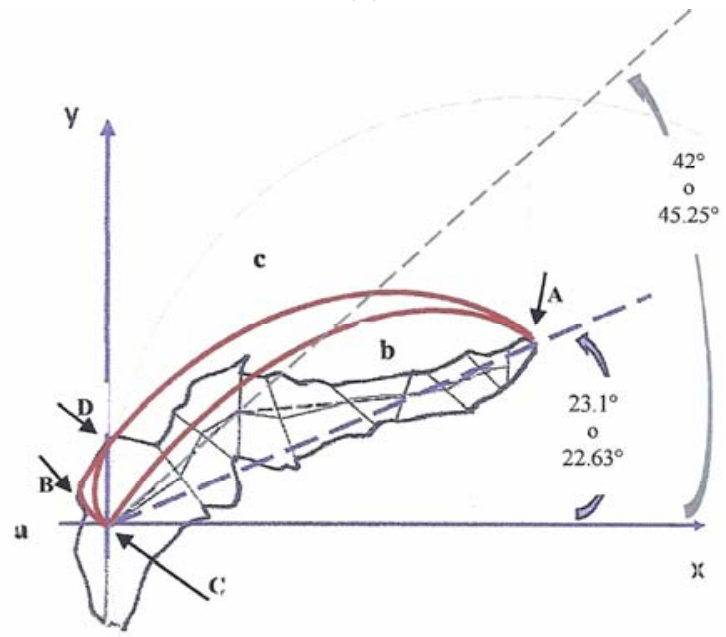

(b)

Figure 5. (a) Real image of a left ulna bone curve fracture, (b) propagation direction of the curve fracture after vectorization. 
In order to obtain propagation direction of curve fracture from flat image, Figure 5(b), values taken in situ of ulna bone are used: $a=0.2631 \mathrm{~cm}$, $b=1.6052 \mathrm{~cm}, c=1.6842 \mathrm{~cm}$, based on trigonometric relations, $s=1.7762 \mathrm{~cm}$. If $C$ is the point of origin of the fracture and $A$ the end point, the value of curve angle obtained for point $A$ is $23.1^{\circ}$. That obtained through Equation (3.1), is $22.63^{\circ}$, showing a difference of only $0.47^{\circ}$. The value of angle measured from the starting point of fracture $C$ is $42^{\circ}$, which compared to $45.25^{\circ}$, results in Equation (3.3), with a difference of $3.25^{\circ}$.

Before knowing under which compression load the curve fracture was generated, it must be taken into account that the finite element models in bone strength are complex, refined and have low probability to become clinically useful in patients both in the near and distant future. For this reason, classical formulation of traditional mechanics shall be used. Since the minimum transversal section of an adult is $6^{*} 10^{-4} \mathrm{~m}^{2}$, the highest compression stress $\sigma$ for adult human bone is $17^{*} 10^{7} \mathrm{Nm}^{-2}$. This is strength per surface unit produced by the curve fracture. Total strength is found after multiplying it by the area of the transversal section of the bone, that is,

$$
F=\sigma_{c} A=\left(17^{*} 10^{7} \mathrm{Nm}^{-2}\right)\left(6^{*} 10^{-4} \mathrm{~m}^{2}\right)=1,020 \mathrm{~N} \text { or } 1,020 \mathrm{Kg} \text { mass. }
$$

Strength, obtained in this way, is approximately 15.7 times the weight of a $65 \mathrm{~kg}$ person, but is higher if the person falls with the speed of his/her own weight when stumbling and falling rigidly. In order to understand this value, we must take into account that a standing person makes a pressure of $15,000 \mathrm{~Pa}$ on the ground.

In order to see strain, let us start with the known value of Young's module under compression in a bone along its axis: $E=0.9^{*} 10^{10} \mathrm{Nm}^{-2}$, for which $E=\frac{\sigma}{\epsilon}$, with $E$ being the longitudinal elasticity modulus, $\sigma$ pressure on the area of the transversal section of the bone, and $\epsilon$ unit strain 
on any point of ulna bone. Therefore, $\epsilon=\frac{\sigma}{E}=\frac{17 * 10^{7} \mathrm{Nm}^{-2}}{9 * 10^{9} \mathrm{Nm}^{-2}}=0.0189$.

This value is dimensionless and means that length of the ulna bone has been reduced approximately $1.89 \%$, that is, since maximum length of left ulna bone in a person with such weight is $210.5 \mathrm{~mm}$ after fracture, this has been reduced $3.97 \mathrm{~mm}$.

The bone has a length $l$ and is an elliptic transversal section, Figure 3. If we make an analogy with a rectangular bar, we know that on the left half of the bone there are a vertical force of the left support and a certain weight, which are equal, in the opposite direction. These forces have different action lines and make a pair which tend to make the ulna bone to turn clockwise. If the top of the ulna bone is compressed, the bottom is subject to traction, which means that the strength of the bone depends on its elastic properties (of which there are not many in a bone). Thus, the internal and external surfaces of the ulna bone are the ones distorted the most, which means that the highest internal strengths will appear on a given point. Therefore, without wide bones, no high moments may be achieved with small internal strengths, and no heavy loads are supported. For this reason when the ulna bone bends with a curvature radius $R$, the internal moment $\tau$ of the bone is given by

$$
\tau=E \frac{I_{y_{1}}}{R}
$$

With $E$ being Young module for bone, $R$ being the curvature radius, and $I_{y_{1}}$ being the moment of inertia of transversal section. Considering ulna bone as a hollow cylinder, Figure 3, $I_{y_{1}}$ is calculated substituting in Equation (9) average values of ulna bone: $x_{1}=2.5 \mathrm{~cm}, x_{2}=1.9 \mathrm{~cm}, y_{1}=1.8 \mathrm{~cm}$, $y_{2}=1.2 \mathrm{~cm}$ with thickness $e=0.3 \mathrm{~cm}$.

Substituting this value in Equation (9), we obtain that internal moment $\tau$ of ulna bone is $\tau=9 * 10^{7} \mathrm{Nm}^{-2} * \frac{0.5535 \mathrm{~cm}^{4}}{0.75 \mathrm{~cm}}=9 * 10^{7} \mathrm{Nm}^{-2}$ $* 0.738 \mathrm{~cm}^{3}=6,642 * 10^{3} \mathrm{Nm}^{-2}$. 
It must be taken into account that inertia is interpreted analogical to the concept of the mass in uniform straight movement, and reflect distribution of the mass of the bone regarding its turning axis. It depends exclusively on the geometry of the bone and the position of the turning axis and not on the forces involved in the movement. For this reason, the higher the distance between the mass and the rotation center, the higher its value. Again, let $R$ be the radius of the cubits and $e$ its thickness, the relationship between the highest tensions on the transversal section of the ulna bone, and the tensile stress applied on such section, the torsional moment of the hollow elliptic section $W_{y}$.

\subsection{Fractal construction of elliptic fractal}

Once the path and propagation of the bone fracture is known, an $\mathrm{X}$-ray image is vectorized and its outline is drawn. Then, a line running along half the outline is drawn, which shall create a sequence of straight line sets that can be numbered. Such polygon shall create an elliptic fractal. With this isolated elliptical fractal, the behaviour pattern of its path is identified, that is, the generator. The curve showing the direction of fractal propagation is isolated and a straight line is drawn from the starting point to its final point, verifying that the pattern or generator is replicated along the elliptical fractal. Its scale factor is calculated, which does not necessarily decrease along its path. It is important to know number, type and shape of inner triangles of the elliptic fractal, as well as the propagation angle of the fractal.

Knowing the behaviour of the generator, it is replicated on every side, at least three or four times, which shall create the real outline of the fracture of ulna bone. Then, if you want to know the future path of the fracture, the generator, at a scale, must be placed on the tip of the fissure and the direction towards which it will go will be known. 


\subsection{Fractal dimension of elliptic fracture}

There are various methods to calculate the fractal dimension, $D_{f}$, these being: Box dimension [10], perimeter-area dimension [12, 13], information dimension [10, 14], mass dimension [10], and ruler dimension [15]. Since these theoretical developments are already known and published, their theoretical development is not shown, but due to their simplicity, we shall briefly review the methods estimating how much space is used by the fractal structure of the element. First, an arbitrary mesh is built on the element to be measured, and the boxes defined by the mesh and occupied by the fractal structure are counted. The process is repeated with meshes with lengths of $n$-th of the size of the previous one, with a decreasing coefficient of boxes, rotating at $x$-degrees. In our case, a 15 degrees rotation increment was used with a 2.0 decreasing coefficient. While the process may be carried out indefinitely, using smaller meshes, the final mesh is delimited by a starting given value of the process. For every mesh and/or size of the box, there is a number of busy boxes, which are tabulated. Tabulated data are graphed in a log-log graph, which means the $x$-axis includes the logarithmic value for size of the box and the $y$-axis includes the logarithmic value for busy boxes. A linear regression is adjusted to such data, in order to know the slope of the straight line, which is used to calculate the fractal dimension.

Table 1 shows values found, in accordance with the calculation method, for every stroke of bone fracture. The table contains six columns: The first one records the theoretical calculation method of fractal dimension or $D_{f}$, the second one contains the fractal's status; the third column contains the fractal's status value of fractal dimension; the fourth column contains standard deviation, $\sigma$, of the straight line with $D_{f}$ slope shown in the fifth column; and the sixth column shows the original stroke of the fracture and statuses $I_{0}, I_{1}, I_{2}, I_{3}$, as well as the total fractal stroke. Values thus obtained have been proven using software Benoit 1.3 [16]. It must be taken into account that $\sigma$ measures probability that an observation is at a certain distance from average observation, and is valid if the system analyzed is a random one. 
Table 1. Parameters of fractal dimension of ulna bone fracture

\begin{tabular}{|c|c|c|c|c|c|}
\hline $\begin{array}{l}\text { Calculation } \\
\text { method }\end{array}$ & Status & $\begin{array}{c}\text { Fractal } \\
\text { dimension } \\
D_{f}\end{array}$ & $\begin{array}{c}\text { Standard } \\
\text { deviation } \\
\sigma\end{array}$ & $\begin{array}{c}\text { Equation of the } \\
\text { straight line } \\
\text { with slope } D_{f}\end{array}$ & Stroke and fractals \\
\hline \multirow{6}{*}{$\begin{array}{c}\text { Box } \\
\text { dimension }\end{array}$} & $\begin{array}{c}\text { Original } \\
\text { stroke }\end{array}$ & 1.95273 & 0.013561 & $3.43 E+06^{*} \mathrm{x}^{-1.95}$ & \multirow{12}{*}{ Original stroke } \\
\hline & $I_{0}$ & 1.95323 & 0.0136908 & $3.43 E+06^{*} \mathrm{x}^{-1.95}$ & \\
\hline & $I_{1}$ & 1.95320 & 0.0136885 & $3.43 E+06^{*} \mathrm{x}^{-1.95}$ & \\
\hline & $I_{2}$ & 1.95319 & 0.0136881 & $3.43 E+06^{*} \mathrm{x}^{-1.95}$ & \\
\hline & $I_{3}$ & 1.95318 & 0.0136862 & $3.43 E+06^{*} \mathrm{x}^{-1.95}$ & \\
\hline & $\begin{array}{l}\text { Total } \\
\text { fractal }\end{array}$ & 1.95294 & 0.0136116 & $3.43 E+06^{*} x^{-1.95}$ & \\
\hline \multirow{6}{*}{$\begin{array}{l}\text { Perimeter- } \\
\text { Area } \\
\text { dimension }\end{array}$} & $\begin{array}{c}\text { Original } \\
\text { stroke }\end{array}$ & 1.02425 & 0.01923234 & $0.0972^{*} x^{1.95}$ & \\
\hline & $I_{0}$ & 1.02399 & 0.1929070 & $0.097^{*} x^{1.95}$ & \\
\hline & $I_{1}$ & 1.02400 & 0.192845 & $0.097^{*} \mathrm{x}^{1.95}$ & \\
\hline & $I_{2}$ & 1.02400 & 0.198322 & $0.097^{*} \mathrm{x}^{1.95}$ & \\
\hline & $I_{3}$ & 1.02402 & 0.198244 & $0.097^{*} \mathrm{x}^{1.95}$ & \\
\hline & $\begin{array}{l}\text { Total } \\
\text { fractal }\end{array}$ & 1.02506 & 0.1937561 & $0.0978^{*} x^{1.95}$ & \\
\hline
\end{tabular}


Table 1. (Continued)

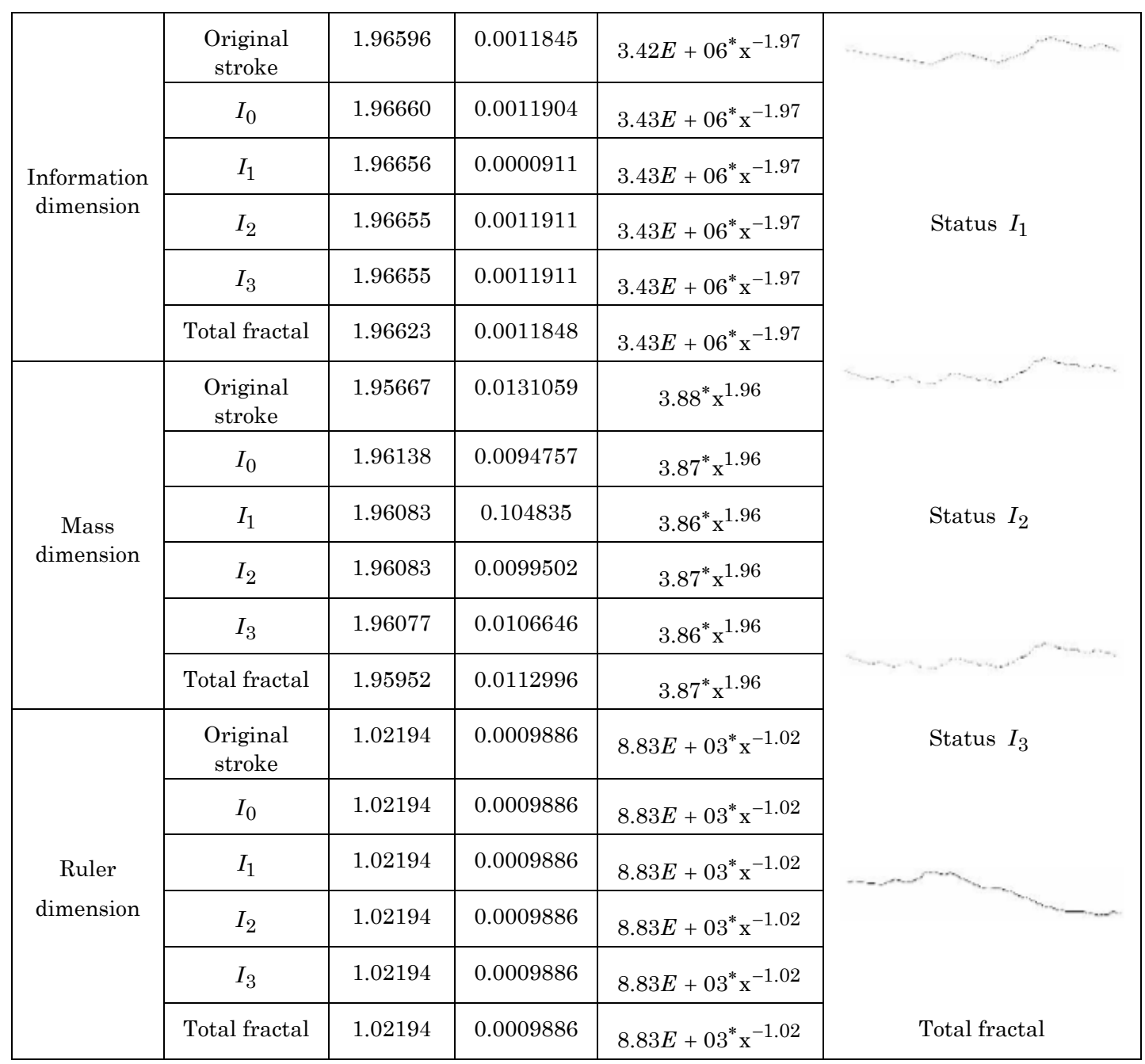


If we take $D_{f t o}$ as the fractal dimension of the original fracture stroke and $D_{f t}$ as the fractal dimension of the total fractal, its difference in absolute value, under the calculation method rule dimension, is zero. The highest difference is seen under the method Mass Dimension, this being 0.00285 .

\section{Methodology}

The methods, or set of procedures determining the research, in accordance with the theoretical description shown, may be summarized in the following steps: (i) obtaining the real image of the ulna bone's elliptic fracture; (ii) vectorization of the fracture, construction of the fractal curve generator and definition of the number of inner triangles creating the elliptic fractal; (iii) definition of the fracture's path direction; (iv) calculation fractal geometry parameters such as number of iterations, reduction factor to be applied, meshing, curve angles; (v) calculation of mechanics of fracture parameters such as: compression stress, strength and moment of inertia of bone transversal section, among many others; (vi) obtaining behaviour patterns; (vii) calculation of elliptic fracture's fractal dimension; and (viii) considering their future paths.

\section{Discussion of Results and Conclusions}

Biomechanical analyses of a bone give us the idea of kinematic and mechanical phenomena shown by live beings, considered as complex systems created by tissues, solids and mechanical bodies. Thus, interest focuses on movement, balance, physics, resistance and injury mechanisms that may happen on the human body as a consequence of certain physical actions. But, why is it important to calculate the fractal index of an injury path in bones, particularly the ulna bone? The answer is rather simple, since the index is related to bone strength and the path of the injury allows knowing its possible propagation speed. The fractal path of the injury may seem, at first sight, chaotic, but that non-linearity possibility allows it to adapt when changes occur. 
A method for biomechanical and fractal analysis of geometrical or polygonal discontinuity has been developed, which creates the sequence of the set of straight lines that may be numbered and identify its path behaviour pattern. Identification of the path is basic in defining the fractal generator or starting status, since it is based on it, it may be defined when a mirror behaviour in one of its sections occurs. Calculation of the scale factor in its behaviour allows considering a future propagation of the fractal, if stresses and moments continue to show in the element under study. This future propagation of the fissure is carried out placing the generation at the corresponding scale, at the final part of the fissure. I think it must be clear that the application of a possible mirror behaviour, in some section of the path, changes fractal generator and, in certain situation, may change future propagation of the fractal as well.

In a similar manner, calculation of propagation angle may change, though the scale factor may not. Calculation of angles in the fissure will be carried out, in our case, through spherical trigonometry, since the fissure is present in such a transversal section.

The fractal shall be manually meshed for calculation of fractal dimension and the results shall be compared to those obtained through commercial software, as it has been carried out here, even though only results obtained with commercial software are shown.

Notice that the elliptical generator obtained here contains three line segments, and, from a theoretical point of view, the axial symmetry property of the generating curve proves affinity of the triangles created by such with the starting status, Figure 4 . To characterize the generating curve as a sequence of sets that may be numbered allows proving the existence of the transformation to a linear affinity of such. In order to help understanding the development of this research easily, a brief list of the methods or set of procedures allowing the replication of what has been done has been included. 


\section{Acknowledgements}

This document was developed with part of the time devoted to the IPN 20120585 research project.

\section{References}

[1] D. Guede, P. González and J. R. Caeiro, Biomecánica y hueso (1): Conceptos básicos y ensayos mecánicos clásicos, Rev. Osteoporos. Metab. Miner. 5(1) (2013), 43-50. ISSN: 1889-836X. http://dx.doi.org/10.4321/S1889-836X2013000100008

[2] J. Ríos-Díaz, J. R. Caeiro Rey, J. J. Martínez Payá, S. Dapía Robleda and M. E. Del Baño Aledo, Relación entre las variables morfo-estructurales de dimensión fractal, lacunaridad y entropía en el hueso trabecular de la porción proximal del fémur, Revista Española de Enfermedades Metabólicas Oseas. 18(01) (2009). Doi:10.1016/S1132-8460(09)70764-0.

[3] Khaled Harrar and Hamami Latifa, The fractal dimension correlated to the bone mineral density, Journal Wseas Transactions on Signal Processing 4(3) (2008). ISSN: 1790-5052. E-ISSN: 2224-3488.

[4] Sanjay Mishra, Andrea E. Tami and Melissa L. Knothe, Fractal Analysis of Bone Cell Syncytium in Normal and Diseased Bone, 2003 Summer Bioengineering Conference, June 25-29, Sonesta Beach Resort in Key Biscayne, Florida. USA.

www.tulane.edu/ sbc2003/pdfdocs/0293.PD

[5] Hyeon-Ju Kang, Song-Wha Jeong, Bong-Hye Jo, Yong-Deok Kim and Seong Sik Kim, Observation of trabecular changes of the mandible after orthognathic surgery using fractal analysis, Journal of the Korean Association of Oral Maxillofacial Surgeons 38 (2012), 96-100. P-ISSN: 2234-7550. E-ISSN: 2234-5930.

http://dx.doi.org/10.5125/jkaoms.2012.38.2.96

[6] S. B. Wells Webster, Nueva Trigonometría Plana y Esférica, D. C. HEATH \& Cía., Editors. Boston, 1917.

http://www.biblioises.com.ar/Contenido/500/520/Astrometria.pdf

[7] Berrosco Manuel, Ramírez María Eva, Enríques-Salamanca José Manuel and PérezPeña Alejandro, Notas y Apuntes de Trigonometría Esférica y Astronomía de Posición, Laboratorio de Astronomía y Geodesia, Departamento de Matemáticas, Facultad de Ciencias, Universidad de Cádiz. España, 2003.

[8] H. O. Peitgen, H. Jürgen and D. Saupe, Fractals for the Classroom, Introduction to Fractals and Chaos, Ed. Springer-Verlag, Second Edition, Part Two, New York, USA, 1993. ISBN: 3-540-97041-X.

[9] V. Talanquer, Fractus, fracta, fractales, Fractales de laberintos y espejos, Fondo de Cultura Económica, Núm. 147(2a), Edición, México, 2002. ISBN: 968-16-6367-4. 
[10] B. B. Mandelbrot, Multifractals and 1/f Noise, Springer-Verlag, 1999. ISBN: 0-38798539-5.

[11] F. Casanova del Angel and J. Retama Velasco, Fractal cracks propagation in aluminum, Modeling and Numerical Simulation of Material Science 3(3A) (2013), 23-32. Doi: 10.4236/mnsms.2013.33A004.

[12] A. R. Imre and J. Bogaert, The fractal dimension as a measure of the quality of habitats, Acta Biometrica 52 (2004), 41-56; Kluwer Academic Publishers, Printed in the Netherlands.

[13] Duanwen Shi, Jian Jiang, Enke Tian and Chiwei Lung, Perimeter-area relation and fractal dimension of fracture surfaces, Journal of Materials Science \& Technology 13(5) (1997), 443-445. ISSN: 1005-0302.

[14] A. Annadhason, Methods of fractal dimension computation, International Journal of Computer Science and Information Technology \& Security 2(1) (2012), 166-169. ISSN: 2249-9555

[15] Bourke Paul, Fractal Dimension Calculator, 2003.

http://palubourke.net/fractals/fracdim/

[16] Benoit 1.3. (2008). TruSoft Int'l Inc. USA. www.trusoft.com 